\title{
State-of-the-art narrative review: multimodality imaging in electrophysiology and cardiac device therapies
}

\author{
Balint Laczay, Divyang Patel, Richard Grimm, Bo Xu \\ Heart, Vascular \& Thoracic Institute, Cleveland Clinic Foundation, Cleveland, OH, USA \\ Contributions: (I) Conception and design: B Xu, D Patel, B Laczay; (II) Administrative support: B Xu, R Grimm; (III) Provision of study materials or \\ patients: All authors; (IV) Collection and assembly of data: B Laczay; (V) Data analysis and interpretation: B Laczay, B Xu; (VI) Manuscript writing: \\ All authors; (VII) Final approval of manuscript: All authors. \\ Correspondence to: Bo Xu, MD, FACC, FRACP, FASE. Section of Cardiovascular Imaging, Robert and Suzanne Tomsich Department of \\ Cardiovascular Medicine, Sydell and Arnold Miller Family Heart, Vascular and Thoracic Institute, Cleveland Clinic, 9500 Euclid Avenue, Desk J1-5, \\ Cleveland, OH 44195, USA. Email: xub@ccf.org.
}

\begin{abstract}
Cardiac electrophysiology procedures have evolved to provide improvement in morbidity and mortality for many patients. Cardiac resynchronization therapy (CRT), implantable cardioverter/defibrillator (ICD) placement and lead extraction procedures are proven procedures, associated with significant reductions in patient morbidity and mortality as well as improved quality of life. The applications and optimization of these therapies are an evolving field. The optimal use and outcomes of cardiac electrophysiology procedures require a multidisciplinary approach to patient selection, device selection, and procedural planning. Cardiac imaging using echocardiography plays a key role in selection of patients for CRT therapy, for guidance of left ventricular (LV) lead placement, and for optimization of atrioventricular pacing delays in patients with CRT. Cardiac computed tomography (CT) is an important tool in assessment of lead perforation, as well as assessing risk of lead extraction and procedural planning. Cardiac magnetic resonance imaging (MRI) is an important adjunct to transthoracic echocardiography for patient selection and risk stratification for defibrillator therapy for multiple disease states including ischemic cardiomyopathy, hypertrophic cardiomyopathy, cardiac sarcoidosis, and arrhythmogenic right ventricular cardiomyopathy (ARVC). Cardiac positron emission tomography (PET) is a useful adjunct to the diagnosis of device infections as well as inflammatory conditions including cardiac sarcoidosis. Our review attempts to summarize the contemporary roles of multimodality imaging in CRT therapy, ICD therapy and lead extraction therapy.
\end{abstract}

Keywords: Echocardiography; cardiac magnetic resonance imaging (cardiac MRI); positron emission tomography/ computed tomography (PET/CT); cardiac resynchronization therapy (CRT); implantable cardioverter/defibrillator (ICD); lead extraction; resynchronization

Submitted Aug 18, 2020. Accepted for publication Nov 30, 2020.

doi: $10.21037 / \mathrm{cdt}-20-724$

View this article at: http://dx.doi.org/10.21037/cdt-20-724

\section{Introduction}

The fields of cardiac electrophysiology (EP) and cardiac imaging have undergone transformative changes with advances in medical technology. The application of cardiac imaging to guiding patient selection in EP as well as risk stratifying and performing EP procedures is a burgeoning field. A wide array of imaging techniques and expertise is required to bridge the gap between two rapidly changing and exciting subspecialties of cardiology. The goal of our paper is to broadly review the application of cardiac imaging in three aspects of EP: cardiac resynchronization therapy (CRT), Lead Extraction, and implantable cardioverter/defibrillator (ICD) placement. For the purposes of this review, we considered English language publications from the years 1990 to 2020, including randomized clinical trials, as well 
as prospective and retrospective cohort studies which were identified using PubMed. We present the following article in accordance with the Narrative Review reporting checklist (available at http://dx.doi.org/10.21037/cdt-20-724).

\section{Role of cardiac imaging in CRT}

CRT is an established therapy for patients with symptomatic heart failure, associated with reduction in cardiovascular mortality and heart failure hospitalizations $(1,2)$. Major society guidelines recommend CRT therapy for patients who have reduced left ventricular ejection fraction (LVEF), a wide QRS ( $\geq 130 \mathrm{~ms}$, preferably in a left bundle branch block pattern) and symptomatic heart failure $(3,4)$. These recommendations stem from the inclusion criteria of major CRT trials, which included patients with LVEF <35\%, QRS $\geq 130 \mathrm{~ms}$, and New York Heart Association (NYHA) class II-IV congestive heart failure (5). Despite positive results in randomized clinical trials, there is a heterogeneity in the effect of CRT therapy on patients. A significant proportion of patients do not benefit less than expected from CRT therapy (6). In a cohort of 302 patients, $43 \%$ did not achieve their definition of success, which was a reduction in the left ventricular end systolic volume (LVESV) of $15 \%$ or more (7). In that cohort, this magnitude of change in LVESV was associated with improved patient outcomes, such as reduced mortality, decreased need for heart transplantation, and less frequent hospitalizations for heart failure (7). The variability in outcomes after CRT placement has resulted in a growing role for multiple imaging modalities for optimizing patient selection and procedural planning. Echocardiography imaging with strain provides additive value through the assessment of mechanical dyssynchrony (8) and guidance of LV lead placement (9) as well as post-placement optimization of CRT therapy with AV delay optimization $(8,10)$. Table 1 shows the relative advantages and disadvantages of echocardiography in electrophysiology procedures.

\section{Mechanical and electrical dyssynchrony}

Improved mechanical and electrical synchrony is one of the proposed mechanisms of action of CRT therapy (5). Left ventricular (LV) dyssynchrony is a frequent finding in heart failure patients and causes worsening myocardial pump function. Abnormalities in cardiac electrical conduction lead to abnormal electrical activation patterns which in turn lead to abnormal contraction of cardiac myocytes (5).
Left bundle branch block, one of the most common forms of electrical dyssynchrony, is present in approximately one third of patients with heart failure, and is associated with increased morbidity, mortality, and sudden cardiac death (SCD) (11). Therefore, the use of echocardiography for defining and quantifying LV dyssynchrony has been investigated in several studies. Dyssynchrony can be classified as atrio-ventricular (between the atria and ventricles), inter-ventricular (between the right and left ventricles), or intra-ventricular (between the different segments of the left ventricle, usually between the septal and lateral wall segments) (12).

Echocardiographic assessment of intra-ventricular dyssynchrony has been shown to have a role in predicting response to CRT. Tissue doppler imaging (TDI) can detect intra-ventricular dyssynchrony, and measuring differences in time to peak myocardial velocity (Ts) in different segments is predictive of CRT response (13). A difference in the basal septal to basal lateral wall Ts of $>60 \mathrm{~ms}$, a difference in opposite wall Ts of $>90 \mathrm{~ms}$, or the maximal difference in Ts $>100 \mathrm{~ms}$ predicted a favorable response to CRT, which was defined as a $>15 \%$ reduction in $L V$ endsystolic volume over a period of 6 months in a cohort of 161 patients (13). The standard deviation of the time-topeak myocardial systolic velocity in $12 \mathrm{LV}$ segments is also a significant predictor of successful response to CRT (14). Speckle tracking strain imaging allows for measurement of myocardial deformation in three different planes: radial, longitudinal, and circumferential. Global longitudinal strain is known to be significantly lower in non-responders compared to responders prior to CRT therapy and increased more in patients who respond to therapy (15). A delay in anteroseptal and posterior segment time-to-peak strain in the radial plane greater than $130 \mathrm{~ms}$ was predictive of CRT response in a group of 161 patients followed over 6 months (13). An increase in the standard deviation of the mean time-to-peak strain in a six-segment model $>76 \mathrm{~ms}$ was also predictive of CRT response in the radial plane (13). An additional method of quantifying dysfunction is the strain delay index, which is defined as the sum of the difference between peak strain and end-systolic strain across all 16 measured segments of the myocardium (16). The difference between peak strain and end-systolic strain was thought to represent "wasted energy" does that does not meaningfully contribute to systole due to the temporal delay in contraction in scarred or fibrotic myocardium. In a cohort of 100 patients who fit criteria for CRT implantation, the presence of a strain delay index greater 
Table 1 The different roles, relative strengths and weaknesses of echocardiography, cardiac CT, CMR, PET CT, SPECT CT imaging in major electrophysiology procedures

\begin{tabular}{|c|c|c|c|}
\hline Imaging modality/application in electrophysiology & Defibrillator therapy & CRT therapy & Lead extraction \\
\hline Advantages: temporal resolution, availability & Assessment of LVEF & $\begin{array}{l}\text { Assessment of dyssynchrony } \\
\text { (TDI, strain imaging) in patient } \\
\text { selection and optimization of } \\
\text { AV delay }\end{array}$ & $\begin{array}{l}\text { Intracardiac } \\
\text { echocardiography for } \\
\text { lead interactions }\end{array}$ \\
\hline
\end{tabular}

Cardiac CT

Advantages: excellent spatial resolution

Disadvantages: radiation exposure, risk of acute kidney injury with contrast enhanced studies

Cardiac MRI Advantages: excellent tissue resolution, cardiac
structural and function assessment

Disadvantages: claustrophobia, relatively contraindicated in advanced renal disease, imaging may be degraded in patients with prior ICDs

PET CT

Advantages: detection of inflammation

Disadvantages: availability, spatial and temporal resolution, cost, radiation exposure

\section{SPECT CT}

Advantages: detection of viability and scar

Disadvantages: radiation exposure, spatial and temporal resolution
Assessment of scar burden in ischemic and non-ischemic cardiomyopathies

Assessment of arrhythmia risk
Delineation of coronary sinus anatomy
Definition of venous anatomy

Risk stratification for extraction procedures
Detection of pocket inflammation

Diagnosis of myocardial inflammation
Detection of pocket inflammation

${ }^{18} \mathrm{~F}-\mathrm{FDG}, 18 \mathrm{~F}$ fluorodeoxyglucose; PET, positron emission tomography; CMR, cardiac magnetic resonance imaging; MRI, magnetic resonance imaging; CT, computed tomography; ICD, implantable cardioverter/defibrillator; TDI, tissue doppler imaging.

than $25 \%$ was shown to predicted reverse remodeling as defined by a $15 \%$ reduction in LVESV with sensitivity and specificity of $95 \%$ and $83 \%$ respectively (17). When applied prospectively in a cohort of 235 patients with both narrow (QRS $<130 \mathrm{~ms}$ ) and wide (QRS $\geq 130 \mathrm{~ms}$ ) complexes and severe $\mathrm{LV}$ dysfunction defined as LVEF $\leq 35 \%$, strain delay index correlated with LVEF improvement $(r=0.45$, $\mathrm{P}<0.001$ ) and using a cutoff value of $>25 \%$ identified $92 \%$ of responders with a positive predictive value of $80 \%$ and negative predictive value of $84 \%$ (17).

The ability of echocardiography to prospectively identify patients with a narrow QRS complex who may benefit from CRT therapy was evaluated in the EchoCRT trial. In this trial of 809 , patients with NYHA class III-IV heart failure, $\mathrm{LVEF}<35 \%$, an indication for defibrillator therapy, a QRS $<130 \mathrm{~ms}$, and evidence of mechanical dyssynchrony on echocardiographic evaluation underwent placement of a CRT-D device. They were then randomized either to receive CRT therapy (CRT-ON) or not to receive CRT therapy (CRT-OFF) (18). The echocardiographic criteria of dyssynchrony in this trial included an opposing-wall delay in the peak systolic velocity of $80 \mathrm{~ms}$ or more in apical 
four-chamber or apical long-axis imaging or a delay in the anteroseptal-to-posterior wall of $130 \mathrm{~ms}$ or more in the mid-LV short-axis view by speckle-tracking radial strain (18). This trial showed excess mortality in the CRT-ON arm (18). Despite the result of this trial, there is still an important role for echocardiography in predicting CRT response.

\section{Guidance of LV lead placement}

The primary therapeutic target of CRT is the restoration of synchronized mechanical contraction in the diseased heart. The restoration of electrical synchrony by placement of a $\mathrm{LV}$ lead is important in achieving this aim. The impact of placing a LV lead on LV hemodynamics can vary significantly based on the location of the lead (19). One study showed up to an $81 \%$ difference in the maximal rate of LV pressure change when systematically testing 11 pacing sites within the left ventricle (12). Radial strain analysis can be used to provide guidance for LV lead placement, as evaluated in the randomized, multi-center TARGET trial (20). In this trial, 220 patients eligible for CRT were randomized to either receive standard CRT placement or CRT placement with echocardiographic guidance of LV lead placement (20). The latest segment of myocardial contraction, defined as the segment with the most delayed peak strain from the onset of the QRS duration in both the basal and mid shortaxis imaging on strain imaging, was chosen for LV lead placement (20). For areas of myocardium with less than $10 \%$ deformational strain, the segment was assumed to be scar and was not chosen (20). Echocardiographic guidance led to a $70 \%$ chance of CRT response, compared to $55 \%$ with standard CRT therapy (20). Similarly, the STARTER trial showed that using strain imaging to localize the last activated myocardial segment for LV lead placement resulted in improved rates of survival and freedom from heart failure hospitalization in 187 patients with NYHA class II-IV symptoms (9).

In addition to localizing areas of optimal deformational strain and the region of latest activation, there is evidence that scar burden as assessed by magnetic resonance imaging (MRI) and nuclear myocardial perfusion imaging (MPI) has prognostic value in predicting CRT response $(21,22)$. A single-center study of 213 patients undergoing CRT placement who underwent positron emission tomography (PET) or single-photon emission computed tomography (CT) looked at the correlation between scar burden and CRT response, with scar extent $>22 \%$ being associated with an improved chance of CRT response (23). This study found that most of the LV leads were placed in the lateral wall, and only $11 \%$ of the LV leads had been placed in area of scar (23). In addition to reducing the chances of CRT response, the presence of LV scar has also been implicated in increased arrhythmia risk and ventricular tachycardia (VT) in the CRT population (24). A study of 57 consecutive patients undergoing CRT placement who had SPECT MPI reported that decreased global viability, as well as decreased local viability at the location of the LV lead had increased the risks of post-implantation VT (25). The utility of combining echocardiography with speckle tracking strain and nuclear SPECT imaging was evaluated in a randomized controlled trial of 182 patients (26). These patients were randomized to either routine insertion of $\mathrm{LV}$ lead, or to a multimodality-guided placement targeting the latest activated LV segment which was scar free on nuclear imaging. The rate of clinical non-response was lower in the patients randomized to imaging guided placement versus those randomized to routine care $(26 \%$ vs. $42 \%$, $\mathrm{P}=0.02)$, despite a non-significant difference in the rate of $\mathrm{LV}$ remodeling as measured by echocardiographic parameters (26). Another randomized controlled trial of 102 patients who underwent CRT placement were randomized to multimodality imaging driven $\mathrm{LV}$ lead placement with strain imaging for the detection of latest activating segment, MRI for assessment of scar and CT imaging for assessment of venous anatomy, or routine placement (27). Despite optimal placement of LV lead in $83 \%$ of their patients, the study failed to demonstrate a significant improvement in $\mathrm{LV}$ remodeling (56\% in imaging guided approach vs. $55 \%$ control, $\mathrm{P}=0.96$ ) or in clinical outcomes (27).

\section{Optimization of atrioventricular synchrony}

In addition to evaluating intra-ventricular dyssynchrony, echocardiography also plays a central role in assessing and optimizing atrio-ventricular dyssynchrony (10,28). An early study of 27 patients showed immediate changes in systolic blood pressure parameters with response to changing atrioventricular (AV) delay (28). As such, programming of the optimal AV delay has been intensely studied as a way to maximize the benefit of CRT (10). The effect of varied AV delay can be studied by evaluation of mitral inflow (29) or by left ventricular outflow tract (LVOT) flow and peak aortic flow.

Assessment of mitral inflow through pulse wave Doppler attempts to optimize AVD to improve LV filling in diastole. A relatively short $A V$ delay may result in truncation of the 
A-wave, which atrial systole, while a prolonged AV delay can result in shortening of total diastolic filling time (30). One of the original methods for achieving an optimal AVD was proposed by Ritter et al. aims to maximize diastolic filling without truncating the A-wave (31). A study of 15 patients with dual-chamber pacemakers showed that applying this method of $\mathrm{AV}$ delay optimization increased cardiac output and decreased pulmonary capillary wedge pressure as measured by a Swan-Ganz catheter (30). A simpler, iterative method of optimizing AV delay has been adopted in major clinical trials such a CARE-HF (1) and SMART-AV (32). This approach entails programming a long AV delay and shortening it iteratively by $20 \mathrm{~ms}$ until the A-wave is truncated and then prolonging by 10 $\mathrm{ms}$ to arrive at the longest possible diastolic filling time without truncating the A-wave (31). The SMART-AV trial randomized 980 patients to standard AV delay or AV delay using the above algorithm, and found no statistically significant difference in LVESV at 3 and 6 months (33). A retrospective cohort of 215 patients who underwent CRT placement underwent AV optimization using either Ritter's method or an iterative method showed no mortality benefit, but did show an improvement in NYHA functional status of at least 1 level in $51 \%$ of patients (29).

While Doppler echocardiography of mitral inflow optimizes AV delay with a focus on diastole, methods that evaluate aortic/LVOT outflow focus on optimizing LV function in systole (31). The use of continuous wave Doppler across the aortic valve or the use of pulsed wave Doppler at the level of the LVOT is a widely accepted way of estimating LV stroke volume (34). Testing the AV delay at $200 \mathrm{~ms}$ and decreasing it in $20 \mathrm{~ms}$ intervals allows the clinician to find the interval with maximum aortic or LVOT velocity time integral (VTI) (10). One randomized trial of 40 patients compared programming the AV delay to maximized aortic valve VTI to an empiric programming of $120 \mathrm{~ms}$ (35). The authors found an improvement in LVEF of $7.8 \%$ and $3.4 \%$ respectively at 3 month interval with an improvement of NYHA class by 1.0 and 0.4 points respectively (35). Despite the lack of benefit to all patients receiving CRT in the SMART-AV trial (33), echocardiographic assessment and optimization of CRT therapy still has a role to play in patients who do not have initial response to CRT, which can be as many as $30 \%$ of all patients $(36,37)$. A cohort of 75 patients referred to a multidisciplinary CRT clinic revealed that suboptimal $\mathrm{AV}$ timing was the most common postulated cause of CRT non-response appearing in $47 \%$ of the cohort (38). Optimization of the AV delay using a mitral inflow method that maximizes diastolic filling without truncating the A-wave had a significant improvement in cumulative survival in this cohort (38).

\section{Role of cardiac imaging in lead extraction}

Due to the increased prevalence of cardiac implantable electronic devices (CIEDs) in current practice, extraction of pacemaker and defibrillator leads is becoming a more common procedure (39). It is estimated that as many as $5 \%$ of all implanted leads will be extracted in their lifetime (40). As such, the detection of lead placement complications, lead infections, and assessment of cardiac anatomy for risk stratifying and performing lead extractions has become critical in managing this increasingly common situation.

\section{Assessment of lead placement and risk of perforation}

Although they have proven to have significant mortality benefits, device implantation is not without the risk of complications, including pneumothorax, lead perforation, or delayed complications such as infection, thrombosis, stenosis and failure to pace appropriately (41). While acute perforation is often immediately identified due to cardiac tamponade and hemodynamic instability, late asymptomatic perforation is often detected on cardiac CT. Overall rates of perforation after pacemaker placement and ICD placement range between $0.1-0.8 \%$, and $0.6-5.2 \%$, respectively (42). Cardiac CT offers high spatial resolution for detection of lead perforations, with one case series of 100 consecutive asymptomatic patients showing a rate as high as $15 \%$ (43). The median time since implantation was 43 months in this series, and the perforations detected were of lesser clinical significance (43). There were no significant differences in lead function or lead impedance, or the occurrence of pericardial effusions (43). Special attention should be paid to atrial leads, where the risk of perforation is higher due to the relative thinness of the atrial wall compared to the ventricular wall. The placement of active fixation leads has also been shown to have a higher rate of perforation than passive fixation leads (44). Clinical predictors of significant perforation include older age, female sex and apical location of the right ventricular lead in a series of 3,822 consecutive patients at a large center (45). Cardiac CT is also the optimal imaging modality for localizing lead position in the right ventricle which has a complex geometry (46). 

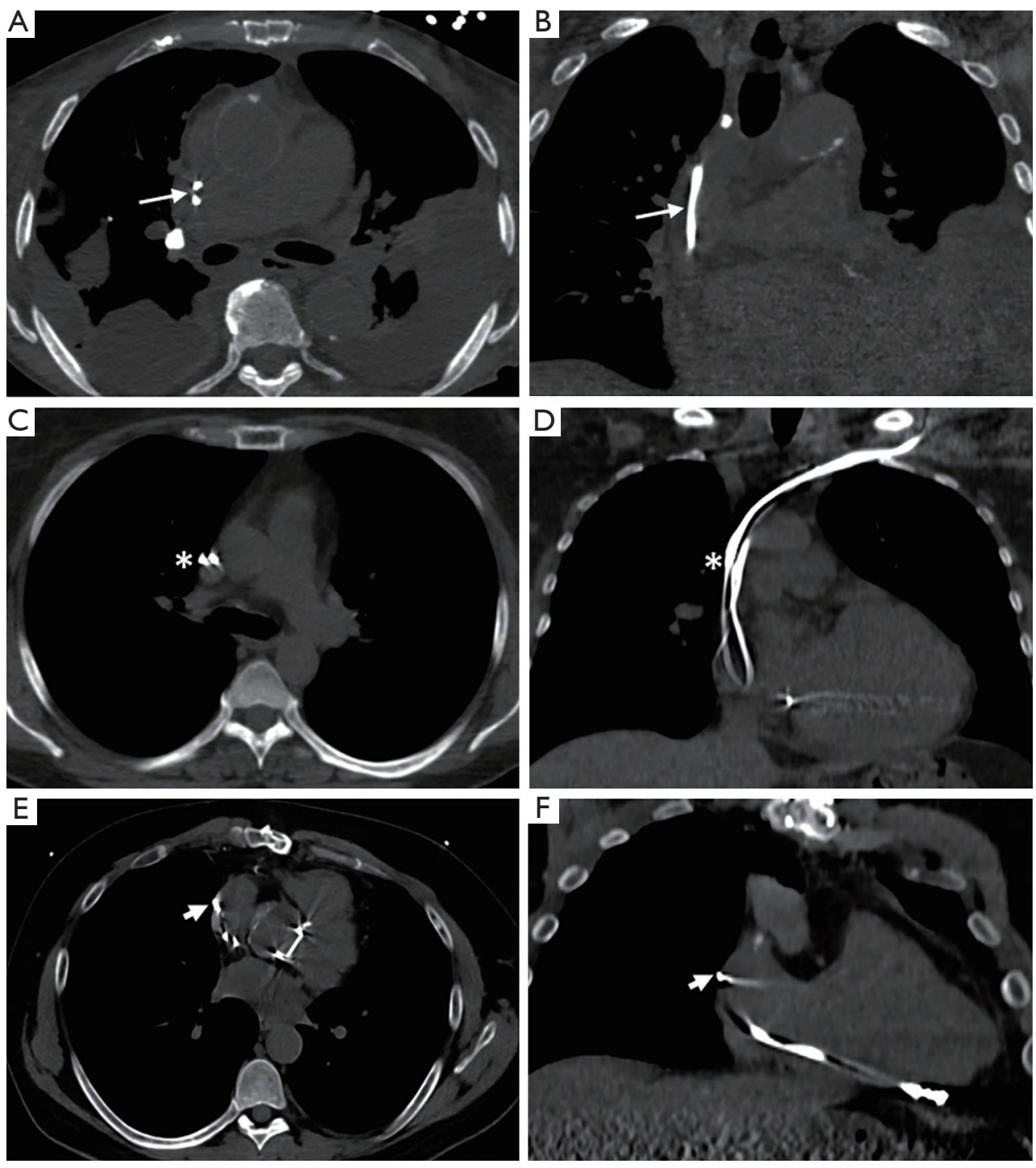

Figure 1 Utility of cardiac computed tomography in planning for device lead extraction. Low risk findings (A: axial image; B: coronal image): all the device leads (arrows) have a central location within the superior vena cava, or are touching less than $1.0 \mathrm{~cm}$ along the length of the superior vena cava. Medium risk findings (C: axial image; D: coronal image): the more lateral device lead is touching the wall of the superior vena cava greater than $1.0 \mathrm{~cm}$ along its length $\left(^{*}\right)$. High risk findings (E: axial image; F: coronal image): the device lead (short arrows) is shown to be partly outside the contour of the superior vena cava.

Figure 1 shows the utility of cardiac CT in assessing lead location and predicting the potential risk of perforation associated with lead removal.

\section{Assessment of lead interactions}

Cardiac CT has also emerged as the leading imaging modality for the planning of lead extraction procedures. A common obstacle to lead extraction procedures is the growth of fibrous tissue and scar around leads, which can cause the formation of lead-lead and lead-vascular interactions (47-49). Defining lead-lead interactions is critical for understanding how removing one lead can affect another, and defining lead-vasculature interactions, especially with regards to the superior vena cava (SVC), is performed by cardiac CT (50). Lead-SVC interactions are of specific importance due to the high morbidity and mortality associated with tears to the SVC as a result of 
complicated lead extractions $(51,52)$. The diagnosis of lead interactions and complex vascular anatomy is crucial, as these cases are more likely to mandate referral to higher volume centers with more procedural experience and better outcomes (53). For example, a cohort of 203 patients undergoing lead extraction with pre-operative CT imaging showed that cases with one lead tip outside the vein contour on imaging required significantly higher procedural time and fluoroscopy time (54). Cardiac CT can define the underlying anatomy of the SVC as well as the presence of vascular adhesions, stenosis and thrombosis. In one study, the presence of vascular adhesions was associated with longer procedural times and the need for larger sheaths to complete extraction (55).

\section{Identifying CIED infection}

Despite improvements in technique and antimicrobial prophylaxis, CIED infections remain a major concern in device therapies (56). The condition carries an in-hospital mortality as high as $29 \%$ (57), and early diagnosis and intervention is associated with improved survival. Patients with infected leads or device infections associated with endocarditis can present with symptoms of frank device erosion, abscess formation and purulent discharge (56). In these patients, the need for extraction is established without the need for advanced imaging $(58,59)$. However, in many patients with only mild, localized infection, adjunctive imaging can be helpful in establishing a diagnosis of CIED associated infection. $18 \mathrm{~F}$ fluorodeoxyglucose $\left({ }^{18} \mathrm{~F}-\mathrm{FDG}\right)$ PET/CT imaging has been utilized in a prospective manner to identify device infection in a cohort of 86 patients with suspected infection with increased FDG activity around the generator pocket suggestive of infection (60) with high diagnostic accuracy (area under curve $=0.98$ ). Once the diagnosis of CIED infection has been confirmed, PET/CT imaging can also serve as an adjunct modality in identifying septic emboli and guiding antibiotic treatment in patients who are unable to undergo cardiac MRI (CMR) due to the presence of their CIED $(61,62)$. A recent meta-analysis of 9 studies addressing the use of ${ }^{18} \mathrm{FDG}$ PET/CT imaging for CIED infection in 208 patients showed that the test has reasonable sensitivity $(72 \%)$ and specificity $(83 \%)$ for the diagnosis (63).

\section{Role of cardiac imaging in defibrillator therapy}

ICD therapy has been a cornerstone therapy for the prevention of SCD for three decades $(64,65)$. The assessment of patients' suitability for ICD therapy depends on their clinical presentation and history as well as parameters found on imaging studies. This evaluation is especially important as low-risk individuals represent a significant portion of total SCD events (66). This has led to the rise of several clinical and objective testing criteria for selecting patients who would benefit from primary prevention ICD placement (67). Several major clinical trials have shown mortality benefit in the placement of primary prevention defibrillators for well-selected patients, and multiple society guidelines have incorporated these studies for guiding clinicians in patient selection $(64,68)$. The use of echocardiography, CMR and cardiac PET/CT are all adjunct imaging modalities in the clinical decision making to undergo ICD placement $(64,69,70)$.

\section{Use of echocardiography for assessment of LV ejection fraction}

Use of echocardiography for evaluation of LV ejection fraction remains of the key imaging parameter for risk stratification for SCD (64). The MADIT-II trial randomized 1,232 patients with a LVEF below $30 \%$ and prior myocardial infarction (MI) to ICD therapy or no ICD therapy, and found a reduction in all-cause mortality $(14 \%$ vs. $20 \% ; \mathrm{P}=0.016$ ) in the ICD therapy (71). Importantly, a substantive $37 \%$ of the patients enrolled in the trial had NYHA class I heart failure, and in the subgroup analysis also benefited from the mortality reduction associated with ICD placement (72). The SCD-HeFT trial enrolled 2,521 patients with LVEF below $35 \%$ and NYHA class II-III symptomatic heart failure (73). It showed that prophylactic ICD placement reduced all-cause mortality ( $22 \%$ vs. $29 \%$; $\mathrm{P}=0.007)$. The MUSTT trial enrolled 704 patients with a LVEF below $40 \%$, non-sustained VT, and inducible VT at electrophysiology study, and showed a decrease in their primary outcome of cardiac arrest or arrhythmic death $(25 \%$ vs. $32 \% ; \mathrm{P}=0.04$ ) (74). Taken together, the results from these trials form the recommendation for many patients with reduced LVEF undergoing defibrillator placement (64). As such, echocardiographic evaluation of LVEF remains paramount for evaluating patients' risk for SCD and need for defibrillator therapy.

\section{Use of CMR for assessment and quantification of scar burden}

Unlike ischemic cardiomyopathy, in which LVEF is one of the major drivers of risk stratification for SCD, non- 
ischemic cardiomyopathy (NICM) often requires adjunctive multimodality imaging for risk stratification (64). The DEFINITE trial randomized 458 patients with NICM with LVEF $35 \%$ and below to ICD therapy or no ICD therapy (75). While there were fewer deaths overall in the group receiving ICDs, the difference was not statistically significant (75). Likewise, the DANISH trial (76) randomized patients with NICM to ICD therapy or usual care and found no significant decrease in all-cause mortality (21\% vs. $23 \%, \mathrm{P}=0.28)$ (76). In population-based studies, as many as $70 \%$ of patients who suffered from SCD had preserved LVEF and would not have been identified as being at risk with assessment of LVEF alone (77). A prospective study that evaluated 137 patients undergoing ICD implantation (78) found a significant increase in the risk of SCD when there was scar detected by MRI exceeding $5 \%$ of the total $\mathrm{LV}$ mass [hazard ratio (HR) $=5.2,95 \%$ confidence intervals (CI): 2.0-13.3]. These findings gave rise to the need to assess the risk of SCD in this patient population with additional methods. Specific conditions including cardiac sarcoidosis, arrhythmogenic right ventricular cardiomyopathy (ARVC) and hypertrophic cardiomyopathy require integration of CMR imaging to appropriately risk stratify patients for defibrillator placement (64).

The presence of late gadolinium enhancement (LGE) on CMR is an important risk marker for SCD in NICM (79). Cardiac sarcoidosis is an etiology of NICM where CMR imaging can help stratify risk of SCD (80). In cardiac sarcoidosis, infiltration of the myocardium by granulomas has been identified as the underlying mechanism for the formation of arrhythmias $(81,82)$. Cardiac manifestations of sarcoidosis include atrioventricular conduction disease, heart failure and ventricular arrhythmias, which are associated with increased risk of mortality $(81,83)$. In about a third of patients, the presenting symptom of cardiac sarcoidosis was ventricular arrhythmia, making early detection and risk prognostication crucial. The presence of LGE identified by CMR) was related to an elevated risk of SCD, ICD discharge and death with a Cox HR of 31.6 (80). This parameter was shown to be superior to LVEF and presentation of heart failure in a cohort of 155 consecutive patients with CMR findings of sarcoidosis (80). A large meta-analysis of 10 studies with 760 patients (84) showed that this association is present both in patients with preserved $(\geq 50 \%)$ and reduced $(\leq 50 \%)$ LVEF. The quantification and distribution of LGE may also impart differential risk of SCD. One study showed that LGE of the right ventricle or LGE involving more than
9 segments of myocardium were prognostic of SCD, while any LGE had far less specificity for predicting SCD (85). The use of CMR to risk stratify patients with cardiac sarcoidosis was incorporated into the latest guidelines for management of ventricular arrhythmias, with cardiac sarcoid patients without prior VT, without prior sudden cardiac arrest, and LVEF greater than 35\% receiving a IIa indication for ICD implantation if there is evidence of extensive scar by CMR or PET imaging (64). PET imaging with ${ }^{18} \mathrm{~F}-\mathrm{FDG}$ imaging has become an important tool in both diagnosis and prognostication in cardiac sarcoidosis (86). The presence of decreased resting perfusion and increased ${ }^{18} \mathrm{~F}$-FDG uptake demonstrates inflammation in the myocardium (86). A large prospective cohort study of 125 patients who underwent PET CT testing (87) showed that the presence of an abnormal perfusion defect and abnormal 18F-FDG signal was associated with an increased risk of major cardiac events after controlling for LVEF (HR: 2.8, 1.03-7.60) (87). Another retrospective cohort of 45 patients found that the presence of myocardial inflammation is related to increased risk of SCD and ICD discharges irrespective of LVEF (88). One advantage of PET imaging over MRI is that it can be used in patients with abnormal renal function as well as in patients who have implanted cardiac devices, which may render highquality CMR imaging in patients with cardiac sarcoidosis difficult (89). Figure 2 shows an example of ${ }^{18}$ F-FDG PET and MRI fusion images diagnostic of cardiac sarcoidosis.

ARVC is another important cause of NICM associated with a significant arrhythmic risk (90). CMR has emerged as an excellent modality for the assessment of right ventricular function, wall motion and quantification of scar burden $(91,92)$. The most common abnormalities found on CMR include dyskinesis of the basal inferior wall of the right ventricle, and basal anterior wall of the right ventricle (93). Abnormalities of the left ventricle can also be encountered, and among them fatty infiltration of the posterolateral $\mathrm{LV}$ wall is most common (93). The presence of CMR abnormalities in addition to electrocardiographic abnormalities in patients who had genetically-confirmed mutations for ARVC led to an increased risk of ventricular arrhythmias in a prospective cohort of 69 patients when compared to patients with only electrocardiographic abnormalities (94). Diffuse involvement and scarring of the right ventricle on CMR is an independent predictor of appropriate ICD shocks in confirmed ARVC patients who have undergone primary prevention defibrillator placement (95).

Hypertrophic cardiomyopathy is a primary genetic disorder causing abnormal fibrosis of cardiac tissue resulting 

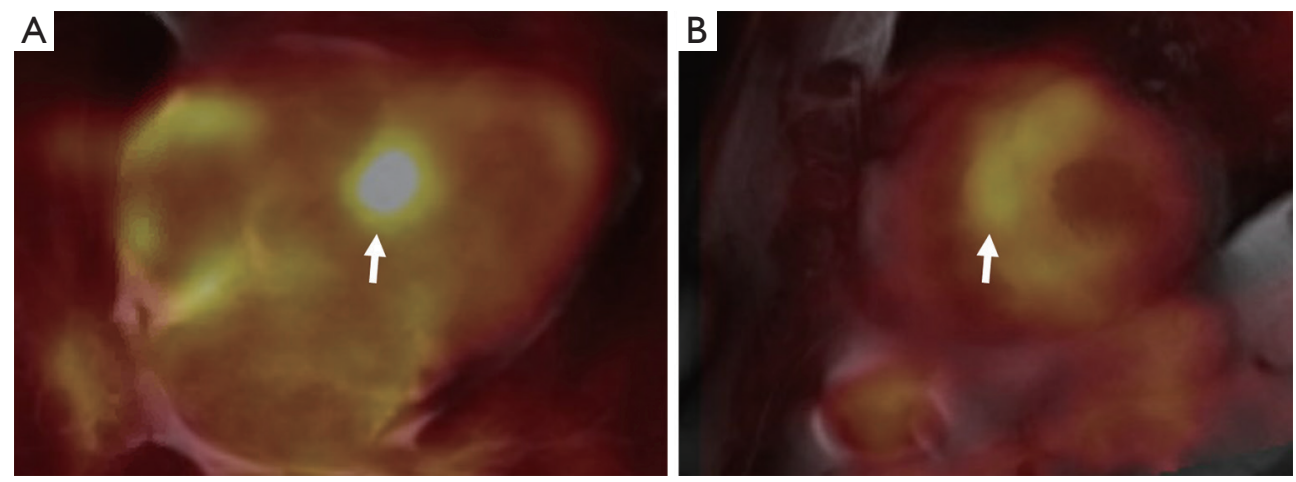

Figure 2 Fusion ${ }^{18}$ F-FDG PET/CMR imaging of a patient with cardiac sarcoidosis. (A) Demonstrates increased ${ }^{18} \mathrm{~F}-\mathrm{FDG}$ uptake in the basal anteroseptum (arrow) on four-chamber imaging, while (B) demonstrates increased ${ }^{18} \mathrm{~F}$-FDG uptake in the entire basal septum (arrow) extending into the basal anterior segment on short-axis imaging. These findings are consistent with active myocardial inflammation. ${ }^{18}$ F-FDG, 18F fluorodeoxyglucose; PET, positron emission tomography; CMR, cardiac magnetic resonance imaging.
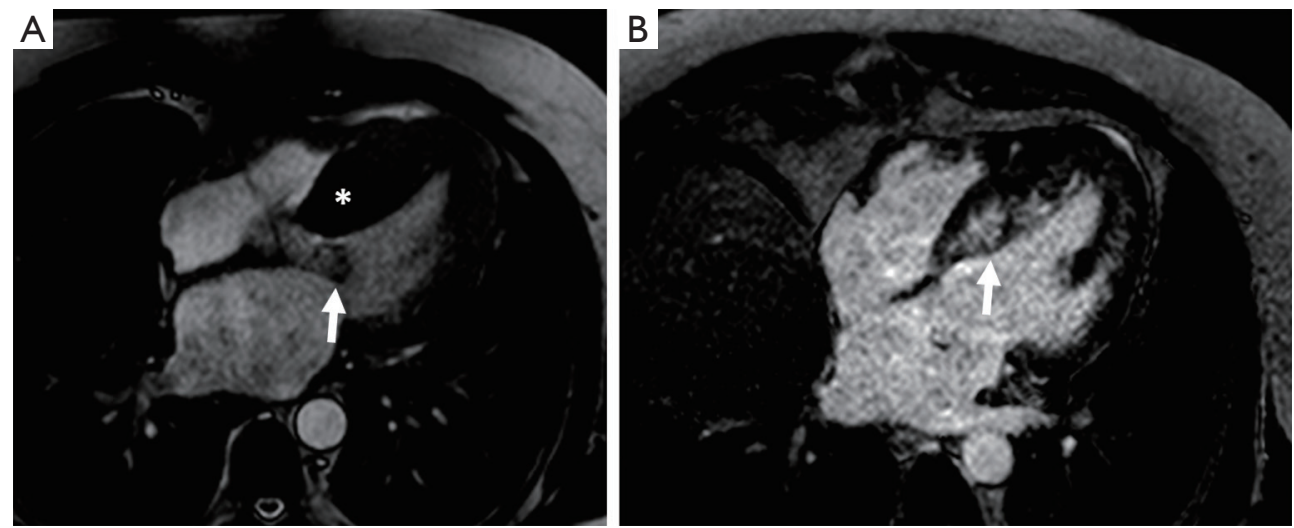

Figure 3 CMR imaging in a patient with hypertrophic obstructive cardiomyopathy. (A) Demonstrates a significantly thickened interventricular septum $\left(^{*}\right)$ on steady-state free precession sequence, associated with marked systolic anterior motion of the mitral valve (arrow). (B) Demonstrates extensive mid-myocardial late gadolinium enhancement, corresponding to the region of increased interventricular septal thickness (arrow). These findings are consistent with extensive scar in a pattern typical for hypertrophic obstructive cardiomyopathy. $\mathrm{CMR}$, cardiac magnetic resonance imaging.

in hypertrophy, cardiac dysfunction, and increased risk of SCD $(96,97)$. Today, it represents the most common cause of SCD in patients under the age of 40 (98), and defibrillator therapy is associated with a decreased risk of SCD in this population. Multiple clinical risk factors are identified for SCD in this population, including prior cardiac arrest, a family history of SCD, LV thickness greater than $30 \mathrm{~mm}$, unexplained syncope, abnormal blood pressure response during exercise and non-sustained VT on telemetry $(48,99)$. The presence of LGE on CMR is now considered a risk-modifier, increasing the risk of SCD when present in this population (100). Typically, LGE in HCM is found in a patchy mid-wall pattern in areas of hypertrophy and at the superior and inferior RV insertion points into the interventricular septum (101). Figure 3 shows one such example of delayed gadolinium uptake. The presence of LGE in HCM has important prognostic implications. A retrospective cohort of 177 patients with CMR data and ambulatory ECG monitoring showed a significant increase in the prevalence of ventricular ectopy and ventricular arrhythmias (PVCs: $89 \%$ vs. $72 \%$; couplets: $40 \%$ vs. $17 \%$; NSVT: $28 \%$ vs. $4 \%$; $\mathrm{P}<0.0001$ to 0.007 ) (102). 
A large meta-analysis with 1,063 patients with hypertrophic cardiomyopathy showed that the presence of LGE had a pooled odds ratio of 2.92 (95\% CI: 1.01-8.42) for cardiac death and a pooled odds ratio of 4.46 (95\% CI: 1.51-13.01) for all-cause mortality (103).

\section{Conclusions}

This review considers several interfaces between the fields of cardiac electrophysiology and cardiac imaging. The use of CRT therapy and ICD therapy has caused significant reductions in mortality and morbidity in many patient subgroups. The appropriate selection, optimization and management of this increasingly large and important patient population requires the cardiologists to be familiar with the roles of various imaging modalities for the comprehensive evaluation of these patients. The development of echocardiography and strain imaging has revolutionized the management and optimization of CRT patients. Likewise, CMR imaging has emerged as a key prognostic tool for the selection of patients appropriate for ICD therapy. The improved anatomic resolution of cardiac CT has led to it being incorporated in the management and selection of patients for lead extraction. Finally, PET CT has a significant role in detecting inflammation, both in inflammatory states such as sarcoidosis and infectious states such as pocket and pacemaker system infections. Despite significant progress in the field, the increased application of multimodality imaging has the potential to increase and apply the benefits of these procedures further, and to a potentially wider patient population. The strengths and weaknesses of each imaging modality for the evaluation of the myocardium allows for a synergistic effect. The superior temporal resolution of echocardiography, the improved anatomic resolution of cardiac CT and the ability of nuclear and MRI imaging to characterize tissue viability and scar have already provided incremental benefit in CRT placement. Likewise, the ability to detect device and pocket infections using multimodality imaging is an evolving field with incremental benefits from multiple imaging modalities, led by PET/CT imaging. As cardiac imaging evolves together with cardiac electrophysiology, the need for clinicians to be familiar with the interface between these two fields will be greater than ever.

\section{Acknowledgments}

Funding: None.

\section{Footnote}

Provenance and Peer Review: This article was commissioned by the editorial office, Cardiovascular Diagnosis and Therapy for the series "Heart Valve Disease". The article has undergone external peer review.

Reporting Checklist: The authors have completed the Narrative Review reporting checklist. Available at http:// dx.doi.org/10.21037/cdt-20-724

Conflicts of Interest: All authors have completed the ICMJE uniform disclosure form (available at http://dx.doi. org/10.21037/cdt-20-724). The series "Heart Valve Disease" was commissioned by the editorial office without any funding or sponsorship. BX served as the unpaid Guest Editor of the series. The authors have no other conflicts of interest to declare.

Ethical Statement: The authors are accountable for all aspects of the work in ensuring that questions related to the accuracy or integrity of any part of the work are appropriately investigated and resolved.

Open Access Statement: This is an Open Access article distributed in accordance with the Creative Commons Attribution-NonCommercial-NoDerivs 4.0 International License (CC BY-NC-ND 4.0), which permits the noncommercial replication and distribution of the article with the strict proviso that no changes or edits are made and the original work is properly cited (including links to both the formal publication through the relevant DOI and the license). See: https://creativecommons.org/licenses/by-nc-nd/4.0/.

\section{References}

1. Cleland JG, Daubert JC, Erdmann E, et al. The effect of cardiac resynchronization on morbidity and mortality in heart failure. N Engl J Med 2005;352:1539-49.

2. Moss AJ, Hall WJ, Cannom DS, et al. Cardiacresynchronization therapy for the prevention of heartfailure events. N Engl J Med 2009;361:1329-38.

3. Yancy CW, Jessup M, Bozkurt B, et al. 2017 ACC/ AHA/HFSA Focused Update of the 2013 ACCF/AHA Guideline for the Management of Heart Failure: A Report of the American College of Cardiology/American Heart Association Task Force on Clinical Practice Guidelines and the Heart Failure Society of America. Circulation 
2017;136:e137-61.

4. Kusumoto FM, Schoenfeld MH, Barrett C, et al. 2018 ACC/AHA/HRS Guideline on the Evaluation and Management of Patients With Bradycardia and Cardiac Conduction Delay: A Report of the American College of Cardiology/American Heart Association Task Force on Clinical Practice Guidelines and the Heart Rhythm Society. J Am Coll Cardiol 2019;74:e51-e156.

5. Linde C, Ellenbogen K, McAlister FA. Cardiac resynchronization therapy (CRT): clinical trials, guidelines, and target populations. Heart Rhythm 2012;9:S3-S13.

6. Sieniewicz BJ, Gould J, Porter B, et al. Understanding non-response to cardiac resynchronisation therapy: common problems and potential solutions. Heart Fail Rev 2019;24:41-54.

7. Ypenburg C, van Bommel RJ, Borleffs CJ, et al. Longterm prognosis after cardiac resynchronization therapy is related to the extent of left ventricular reverse remodeling at midterm follow-up. J Am Coll Cardiol 2009;53:483-90.

8. Spartalis M, Tzatzaki E, Spartalis E, et al. The Role of Echocardiography in the Optimization of Cardiac Resynchronization Therapy: Current Evidence and Future Perspectives. Open Cardiovasc Med J 2017;11:133-45.

9. Saba S, Marek J, Schwartzman D, et al. Echocardiographyguided left ventricular lead placement for cardiac resynchronization therapy: results of the Speckle Tracking Assisted Resynchronization Therapy for Electrode Region trial. Circ Heart Fail 2013;6:427-34.

10. Waggoner AD, de las Fuentes L, Davila-Roman VG. Doppler echocardiographic methods for optimization of the atrioventricular delay during cardiac resynchronization therapy. Echocardiography 2008;25:1047-55.

11. Baldasseroni S, Opasich C, Gorini M, et al. Left bundlebranch block is associated with increased 1-year sudden and total mortality rate in 5517 outpatients with congestive heart failure: a report from the Italian network on congestive heart failure. Am Heart J 2002;143:398-405.

12. Marechaux S, Menet A, Guyomar Y, et al. Role of echocardiography before cardiac resynchronization therapy: new advances and current developments. Echocardiography 2016;33:1745-52.

13. Yu CM, Gorcsan J 3rd, Bleeker GB, et al. Usefulness of tissue Doppler velocity and strain dyssynchrony for predicting left ventricular reverse remodeling response after cardiac resynchronization therapy. Am J Cardiol 2007;100:1263-70.

14. Yu CM, Fung JW, Zhang Q, et al. Tissue Doppler imaging is superior to strain rate imaging and postsystolic shortening on the prediction of reverse remodeling in both ischemic and nonischemic heart failure after cardiac resynchronization therapy. Circulation 2004;110:66-73.

15. To AC, Benatti RD, Sato K, et al. Strain-time curve analysis by speckle tracking echocardiography in cardiac resynchronization therapy: Insight into the pathophysiology of responders vs. non-responders. Cardiovasc Ultrasound 2016;14:14.

16. Lim P, Buakhamsri A, Popovic ZB, et al. Longitudinal Strain Delay Index by Speckle Tracking Imaging. Circulation 2008;118:1130-7.

17. Lim P, Donal E, Lafitte S, et al. Multicentre study using strain delay index for predicting response to cardiac resynchronization therapy (MUSIC study). Eur J Heart Fail 2011;13:984-91.

18. Ruschitzka F, Abraham WT, Singh JP, et al. Cardiacresynchronization therapy in heart failure with a narrow QRS complex. N Engl J Med 2013;369:1395-405.

19. Derval N, Steendijk P, Gula LJ, et al. Optimizing hemodynamics in heart failure patients by systematic screening of left ventricular pacing sites: the lateral left ventricular wall and the coronary sinus are rarely the best sites. J Am Coll Cardiol 2010;55:566-75.

20. Khan FZ, Virdee MS, Palmer CR, et al. Targeted left ventricular lead placement to guide cardiac resynchronization therapy: the TARGET study: a randomized, controlled trial. J Am Coll Cardiol 2012;59:1509-18.

21. Aggarwal NR, Martinez MW, Gersh BJ, et al. Role of cardiac MRI and nuclear imaging in cardiac resynchronization therapy. Nat Rev Cardiol 2009;6:759-70.

22. Ypenburg C, Schalij MJ, Bleeker GB, et al. Impact of viability and scar tissue on response to cardiac resynchronization therapy in ischaemic heart failure patients. Eur Heart J 2007;28:33-41.

23. Xu YZ, Cha YM, Feng D, et al. Impact of myocardial scarring on outcomes of cardiac resynchronization therapy: extent or location? J Nucl Med 2012;53:47-54.

24. Fernández-Armenta J, Berruezo A, Mont L, et al. Use of myocardial scar characterization to predict ventricular arrhythmia in cardiac resynchronization therapy. Europace 2012;14:1578-86.

25. Žižek D, Cvijić M, Ležaić L, et al. Impact of myocardial viability assessed by myocardial perfusion imaging on ventricular tachyarrhythmias in cardiac resynchronization therapy. J Nucl Cardiol 2013;20:1049-59.

26. Sommer A, Kronborg MB, Nørgaard BL, et al. Multimodality imaging-guided left ventricular lead 
placement in cardiac resynchronization therapy: a randomized controlled trial. Eur J Heart Fail 2016;18:1365-74.

27. Borgquist R, Carlsson M, Markstad H, et al. Cardiac Resynchronization Therapy Guided by Echocardiography, MRI, and CT Imaging: A Randomized Controlled Study. JACC Clin Electrophysiol 2020;6:1300-9.

28. Auricchio A, Stellbrink C, Block M, et al. Effect of Pacing Chamber and Atrioventricular Delay on Acute Systolic Function of Paced Patients With Congestive Heart Failure. Circulation 1999;99:2993-3001.

29. Kedia N, Ng K, Apperson-Hansen C, et al. Usefulness of Atrioventricular Delay Optimization Using Doppler Assessment of Mitral Inflow in Patients Undergoing Cardiac Resynchronization Therapy. Am J Cardiol 2006;98:780-5.

30. Ishikawa T, Sumita S, Kimura K, et al. Prediction of optimal atrioventricular delay in patients with implanted DDD pacemakers. Pacing Clin Electrophysiol 1999;22:1365-71.

31. Brabham WW, Gold MR. The role of AV and VV optimization for CRT. J Arrhythm 2013;29:153-61.

32. Ellenbogen KA, Gold MR, Meyer TE, et al. Primary Results From the SmartDelay Determined AV Optimization: A Comparison to Other AV Delay Methods Used in Cardiac Resynchronization Therapy (SMART-AV) Trial. Circulation 2010;122:2660-8.

33. Stein KM, Ellenbogen KA, Gold MR, et al. SmartDelay determined AV optimization: a comparison of AV delay methods used in cardiac resynchronization therapy (SMART-AV): rationale and design. Pacing Clin Electrophysiol 2010;33:54-63.

34. Colocousis JS, Huntsman LL, Curreri PW. Estimation of stroke volume changes by ultrasonic doppler. Circulation 1977;56:914-7.

35. Sawhney NS, Waggoner AD, Garhwal S, et al. Randomized prospective trial of atrioventricular delay programming for cardiac resynchronization therapy. Heart Rhythm 2004;1:562-7.

36. Chung ES, Leon AR, Tavazzi L, et al. Results of the Predictors of Response to CRT (PROSPECT) trial. Circulation 2008;117:2608-16.

37. Abraham WT, Fisher WG, Smith AL, et al. Cardiac resynchronization in chronic heart failure. N Engl J Med 2002;346:1845-53.

38. Mullens W, Grimm RA, Verga T, et al. Insights from a cardiac resynchronization optimization clinic as part of a heart failure disease management program. J Am Coll
Cardiol 2009;53:765-73.

39. Wilkoff BL, Love CJ, Byrd CL, et al. Transvenous Lead Extraction: Heart Rhythm Society Expert Consensus on Facilities, Training, Indications, and Patient Management: This document was endorsed by the American Heart Association (AHA). Heart Rhythm 2009;6:1085-104.

40. Ehieli WL, Boll DT, Marin D, et al. Use of Preprocedural MDCT for Cardiac Implantable Electric Device Lead Extraction: Frequency of Findings That Change Management. AJR Am J Roentgenol 2017;208:770-6.

41. Ellenbogen KA, Wood MA, Shepard RK. Delayed complications following pacemaker implantation. Pacing Clin Electrophysiol 2002;25:1155-8.

42. Khan MN, Joseph G, Khaykin Y, et al. Delayed lead perforation: a disturbing trend. Pacing Clin Electrophysiol 2005;28:251-3.

43. Hirschl DA, Jain VR, Spindola-Franco H, et al. Prevalence and Characterization of Asymptomatic Pacemaker and ICD Lead Perforation on CT. Pacing Clin Electrophysiol 2007;30:28-32.

44. Mahapatra S, Bybee KA, Bunch TJ, et al. Incidence and predictors of cardiac perforation after permanent pacemaker placement. Heart Rhythm 2005;2:907-11.

45. Cano Ó, Andrés A, Alonso P, et al. Incidence and predictors of clinically relevant cardiac perforation associated with systematic implantation of active-fixation pacing and defibrillation leads: a single-centre experience with over 3800 implanted leads. Europace 2017;19:96-102.

46. Moore P, Coucher J, Ngai S, et al. Imaging and Right Ventricular Pacing Lead Position: A Comparison of CT, MRI, and Echocardiography. Pacing Clin Electrophysiol 2016;39:382-92.

47. Epstein LM, Love CJ, Wilkoff BL, et al. Superior Vena Cava Defibrillator Coils Make Transvenous Lead Extraction More Challenging and Riskier. J Am Coll Cardiol 2013;61:987-9.

48. Segreti L, Di Cori A, Soldati E, et al. Major predictors of fibrous adherences in transvenous implantable cardioverter-defibrillator lead extraction. Heart Rhythm 2014;11:2196-201.

49. Schaller RD, Sadek MM, Cooper JM. Simultaneous lead traction from above and below: A novel technique to reduce the risk of superior vena cava injury during transvenous lead extraction. Heart Rhythm 2018;15:1655-63.

50. Holm MA, Vatterott PJ, Gaasedelen EN, et al. Algorithm for the analysis of pre-extraction computed tomographic images to evaluate implanted lead-lead interactions and lead-vascular attachments. Heart 
Rhythm 2020;17:1009-16.

51. Brunner MP, Cronin EM, Wazni O, et al. Outcomes of patients requiring emergent surgical or endovascular intervention for catastrophic complications during transvenous lead extraction. Heart Rhythm 2014;11:419-25.

52. Sood N, Martin David T, Lampert R, et al. Incidence and Predictors of Perioperative Complications With Transvenous Lead Extractions. Circ Arrhythm Electrophysiol 2018;11:e004768.

53. Di Monaco A, Pelargonio G, Narducci ML, et al. Safety of transvenous lead extraction according to centre volume: a systematic review and meta-analysis. Europace 2014;16:1496-507.

54. Patel D, Sripariwuth A, Abozeed M, et al. Lead Location as Assessed on Cardiac Computed Tomography and Difficulty of Percutaneous Transvenous Extraction. JACC Clin Electrophysiol 2019;5:1432.

55. Lewis RK, Pokorney SD, Greenfield RA, et al. Preprocedural ECG-Gated Computed Tomography for Prevention of Complications during Lead Extraction. Pacing Clin Electrophysiol 2014;37:1297-305.

56. Voigt A, Shalaby A, Saba S. Continued rise in rates of cardiovascular implantable electronic device infections in the United States: temporal trends and causative insights. Pacing Clin Electrophysiol 2010;33:414-9.

57. Viganego F, O'Donoghue S, Eldadah Z, et al. Effect of Early Diagnosis and Treatment With Percutaneous Lead Extraction on Survival in Patients With Cardiac Device Infections. Am J Cardiol 2012;109:1466-71.

58. Baddour LM, Epstein AE, Erickson CC, et al. Update on cardiovascular implantable electronic device infections and their management: a scientific statement from the American Heart Association. Circulation 2010;121:458-77.

59. Bongiorni MG, Burri H, Deharo JC, et al. 2018 EHRA expert consensus statement on lead extraction: recommendations on definitions, endpoints, research trial design, and data collection requirements for clinical scientific studies and registries: endorsed by APHRS/ HRS/LAHRS. Europace 2018;20:1217.

60. Ahmed FZ, James J, Cunnington C, et al. Early diagnosis of cardiac implantable electronic device generator pocket infection using 18F-FDG-PET/CT. Eur Heart J Cardiovasc Imaging 2015;16:521-30.

61. Amraoui S, Tlili G, Sohal M, et al. Contribution of PET Imaging to the Diagnosis of Septic Embolism in Patients With Pacing Lead Endocarditis. JACC Cardiovasc Imaging 2016;9:283-90.
62. Bernard L, Dinh A, Ghout I, et al. Antibiotic treatment for 6 weeks versus 12 weeks in patients with pyogenic vertebral osteomyelitis: an open-label, non-inferiority, randomised, controlled trial. Lancet 2015;385:875-82.

63. Wang TKM, Sánchez-Nadales A, Igbinomwanhia E, et al. Diagnosis of Infective Endocarditis by Subtype Using 18 F-Fluorodeoxyglucose Positron Emission Tomography/ Computed Tomography. Circ Cardiovasc Imaging 2020;13:e010600.

64. Al-Khatib SM, Stevenson WG, Ackerman MJ, et al. 2017 AHA/ACC/HRS Guideline for Management of Patients With Ventricular Arrhythmias and the Prevention of Sudden Cardiac Death: A Report of the American College of Cardiology/American Heart Association Task Force on Clinical Practice Guidelines and the Heart Rhythm Society. Circulation 2018;138:e272-e391.

65. Kusumoto FM, Calkins H, Boehmer J, et al. HRS/ ACC/AHA Expert Consensus Statement on the Use of Implantable Cardioverter-Defibrillator Therapy in Patients Who Are Not Included or Not Well Represented in Clinical Trials. J Am Coll Cardiol 2014;64:1143-77.

66. Myerburg RJ, Junttila MJ. Sudden Cardiac Death Caused by Coronary Heart Disease. Circulation 2012;125:1043-52.

67. Poole JE. Present Guidelines for Device Implantation: Clinical Considerations and Clinical Challenges From Pacing, Implantable Cardiac Defibrillator, and Cardiac Resynchronization Therapy. Circulation 2014;129:383-94.

68. Priori SG, Blomström-Lundqvist C, Mazzanti A, et al. 2015 ESC Guidelines for the management of patients with ventricular arrhythmias and the prevention of sudden cardiac death: The Task Force for the Management of Patients with Ventricular Arrhythmias and the Prevention of Sudden Cardiac Death of the European Society of Cardiology (ESC). Endorsed by: Association for European Paediatric and Congenital Cardiology (AEPC). Eur Heart J 2015;36:2793-867.

69. Yancy CW, Jessup M, Bozkurt B, et al. 2013 ACCF/AHA Guideline for the Management of Heart Failure: Executive Summary: a report of the American College of Cardiology Foundation/American Heart Association Task Force on practice guidelines. Circulation 2013;128:1810-52.

70. Russo AM, Stainback RF, Bailey SR, et al. ACCF/HRS/ AHA/ASE/HFSA/SCAI/SCCT/SCMR 2013 appropriate use criteria for implantable cardioverter-defibrillators and cardiac resynchronization therapy: a report of the American College of Cardiology Foundation appropriate use criteria task force, Heart Rhythm Society, American 
Heart Association, American Society of Echocardiography, Heart Failure Society of America, Society for Cardiovascular Angiography and Interventions, Society of Cardiovascular Computed Tomography, and Society for Cardiovascular Magnetic Resonance. J Am Coll Cardiol 2013;61:1318-68.

71. Moss AJ, Zareba W, Hall WJ, et al. Prophylactic Implantation of a Defibrillator in Patients with Myocardial Infarction and Reduced Ejection Fraction. N Engl J Med 2002;346:877-83.

72. Greenberg H, Case RB, Moss AJ, et al. Analysis of mortality events in the Multicenter Automatic Defibrillator Implantation Trial (MADIT-II). J Am Coll Cardiol 2004;43:1459-65.

73. Bardy GH, Lee KL, Mark DB, et al. Amiodarone or an Implantable Cardioverter-Defibrillator for Congestive Heart Failure. N Engl J Med 2005;352:225-37.

74. Buxton AE, Lee KL, Dicarlo L, et al. Electrophysiologic Testing to Identify Patients with Coronary Artery Disease Who Are at Risk for Sudden Death. N Engl J Med 2000;342:1937-45.

75. Kadish A, Dyer A, Daubert JP, et al. Prophylactic Defibrillator Implantation in Patients with Nonischemic Dilated Cardiomyopathy. N Engl J Med 2004;350:2151-8.

76. Kober L, Thune JJ, Nielsen JC, et al. Defibrillator Implantation in Patients with Nonischemic Systolic Heart Failure. N Engl J Med 2016;375:1221-30.

77. Stecker EC, Vickers C, Waltz J, et al. Population-Based Analysis of Sudden Cardiac Death With and Without Left Ventricular Systolic Dysfunction. J Am Coll Cardiol 2006;47:1161-6.

78. Klem I, Weinsaft JW, Bahnson TD, et al. Assessment of myocardial scarring improves risk stratification in patients evaluated for cardiac defibrillator implantation. J Am Coll Cardiol 2012;60:408-20.

79. Kuruvilla S, Adenaw N, Katwal AB, et al. Late Gadolinium Enhancement on Cardiac Magnetic Resonance Predicts Adverse Cardiovascular Outcomes in Nonischemic Cardiomyopathy. Circ Cardiovasc Imaging 2014;7:250-8.

80. Greulich S, Deluigi CC, Gloekler S, et al. CMR Imaging Predicts Death and Other Adverse Events in Suspected Cardiac Sarcoidosis. JACC Cardiovasc Imaging 2013;6:501-11.

81. Yazaki Y, Isobe M, Hiroe M, et al. Prognostic determinants of long-term survival in Japanese patients with cardiac sarcoidosis treated with prednisone. Am J Cardiol 2001;88:1006-10.

82. Reuhl J, Schneider M, Sievert H, et al. Myocardial sarcoidosis as a rare cause of sudden cardiac death. Forensic Sci Int 1997;89:145-53.

83. Kandolin R, Lehtonen J, Airaksinen J, et al. Cardiac Sarcoidosis. Circulation 2015;131:624-32.

84. Coleman GC, Shaw PW, Balfour PC, et al. Prognostic Value of Myocardial Scarring on CMR in Patients With Cardiac Sarcoidosis. JACC Cardiovasc Imaging 2017;10:411-20.

85. Crawford T, Mueller G, Sarsam S, et al. Magnetic Resonance Imaging for Identifying Patients With Cardiac Sarcoidosis and Preserved or Mildly Reduced Left Ventricular Function at Risk of Ventricular Arrhythmias. Circ Arrhythm Electrophysiol 2014;7:1109-15.

86. Skali H, Schulman AR, Dorbala S. 18F-FDG PET/CT for the assessment of myocardial sarcoidosis. Curr Cardiol Rep 2013;15:352.

87. Blankstein R, Osborne M, Naya M, et al. Cardiac positron emission tomography enhances prognostic assessments of patients with suspected cardiac sarcoidosis. J Am Coll Cardiol 2014;63:329-36.

88. Betensky BP, Tschabrunn CM, Zado ES, et al. Longterm follow-up of patients with cardiac sarcoidosis and implantable cardioverter-defibrillators. Heart Rhythm 2012;9:884-91.

89. Birnie DH, Nery PB, Ha AC, et al. Cardiac Sarcoidosis. J Am Coll Cardiol 2016;68:411-21.

90. Groeneweg JA, Bhonsale A, James CA, et al. Clinical Presentation, Long-Term Follow-Up, and Outcomes of 1001 Arrhythmogenic Right Ventricular Dysplasia/ Cardiomyopathy Patients and Family Members. Circ Cardiovasc Genet 2015;8:437-46.

91. Marcus FI, McKenna WJ, Sherrill D, et al. Diagnosis of arrhythmogenic right ventricular cardiomyopathy/ dysplasia: Proposed Modification of the Task Force Criteria. Eur Heart J 2010;31:806-14.

92. Liu T, Pursnani A, Sharma UC, et al. Effect of the 2010 task force criteria on reclassification of cardiovascular magnetic resonance criteria for arrhythmogenic right ventricular cardiomyopathy. J Cardiovasc Magn Reson 2014;16:47.

93. Te Riele AS, James CA, Philips B, et al. Mutationpositive arrhythmogenic right ventricular dysplasia/ cardiomyopathy: the triangle of dysplasia displaced. J Cardiovasc Electrophysiol 2013;24:1311-20.

94. te Riele AS, Bhonsale A, James CA, et al. Incremental Value of Cardiac Magnetic Resonance Imaging in Arrhythmic Risk Stratification of Arrhythmogenic Right Ventricular Dysplasia/Cardiomyopathy-Associated 
Desmosomal Mutation Carriers. J Am Coll Cardiol 2013;62:1761-9.

95. Corrado D, Calkins H, Link MS, et al. Prophylactic Implantable Defibrillator in Patients With Arrhythmogenic Right Ventricular Cardiomyopathy/Dysplasia and No Prior Ventricular Fibrillation or Sustained Ventricular Tachycardia. Circulation 2010;122:1144-52.

96. Elliott PM, Sharma S, Varnava A, et al. Survival after cardiac arrest or sustained ventricular tachycardia in patients with hypertrophic cardiomyopathy. J Am Coll Cardiol 1999;33:1596-601.

97. Elliott PM, Poloniecki J, Dickie S, et al. Sudden death in hypertrophic cardiomyopathy: identification of high risk patients. J Am Coll Cardiol 2000;36:2212-8.

98. Maron BJ, Shen WK, Link MS, et al. Efficacy of Implantable Cardioverter-Defibrillators for the Prevention of Sudden Death in Patients with Hypertrophic Cardiomyopathy. N Engl J Med 2000;342:365-73.

99. Gersh BJ, Maron BJ, Bonow RO, et al. 2011 ACCF/ AHA Guideline for the Diagnosis and Treatment of Hypertrophic Cardiomyopathy: a report of the American College of Cardiology Foundation/American Heart Association Task Force on Practice Guidelines. Developed in collaboration with the American Association for Thoracic Surgery, American Society of Echocardiography, American Society of Nuclear Cardiology, Heart Failure Society of America, Heart Rhythm Society, Society for Cardiovascular Angiography and Interventions, and Society of Thoracic Surgeons. J Am Coll Cardiol 2011;58:e212-60.

100. Steriotis AK, Sharma S. Risk Stratification in Hypertrophic Cardiomyopathy. Eur Cardiol 2015;10:31-6.

101. Rudolph A, Abdel-Aty H, Bohl S, et al. Noninvasive Detection of Fibrosis Applying Contrast-Enhanced Cardiac Magnetic Resonance in Different Forms of Left Ventricular Hypertrophy. J Am Coll Cardiol 2009;53:284-91.

102.Adabag AS, Maron BJ, Appelbaum E, et al. Occurrence and Frequency of Arrhythmias in Hypertrophic Cardiomyopathy in Relation to Delayed Enhancement on Cardiovascular Magnetic Resonance. J Am Coll Cardiol 2008;51:1369-74.

103. Green JJ, Berger JS, Kramer CM, et al. Prognostic Value of Late Gadolinium Enhancement in Clinical Outcomes for Hypertrophic Cardiomyopathy. JACC Cardiovasc Imaging 2012;5:370-7.
Cite this article as: Laczay B, Patel D, Grimm R, Xu B. State-of-the-art narrative review: multimodality imaging in electrophysiology and cardiac device therapies. Cardiovasc Diagn Ther 2021;11(3):881-895. doi: 10.21037/cdt-20-724 